\title{
MODELAGEM NUMÉRICA DA VARIAÇÃO TRIMESTRAL DOS PARÂMETROS FÍSICOS DA VEGETAÇÃO
}

Gabriel Pereira*

Maria Elisa Siqueira Silva**

Resumo: O objetivo do presente trabalho é verificar o impacto da atualização dos parâmetros físico-químicos e biológicos utilizados pelo Biosphere Atmosphere Transfer Scheme (Bats) acoplado ao modelo numérico RegCM4. Na rodada de controle, usando os dados originais do RegCM4, a precipitação para o ano de 2007 foi subestimada em 12\%, apresentando uma correlação de $84 \%$ (significante a $\mathrm{p}<0,05$, teste t-student). Porém, com a modelagem da variação trimestral dos parâmetros físicos, obteve-se uma redução significativa do erro nos valores de precipitação (subestimativa de 3\% e correlação de 92\%, significante a $p<0,05$, teste t-student). Ainda, em relação à temperatura máxima e mínima, percebe-se que ambas as simulações apresentam boa concordância com os dados observados.

Palavras-chave: RegCM4, parâmetros físicos, dinâmica da vegetação, modelagem numérica, sensoriamento remoto.

\section{NUMERICAL MODELING OF TRIMONTHLY VARIATION OF VEGETATION PHYSICAL PARAMETERS}

Abstract: This work aims to evaluate the impact of updating the physical-chemical and biological properties used by the Biosphere Atmosphere Transfer Scheme (Bats) in RegCM4 numerical model. In original simulation, using the original dataset of RegCM4, the precipitation for 2007 was underestimated in $12 \%$, presenting a correlation of $84 \%$ (significant at $p<0.05$, Student's t-test). However, the trimonthly modeling of the physical parameters occasioned a significant reduction of precipitation error (underestimation of $3 \%$ and $92 \%$ correlation, significant at $p<0.05$, Student's $t$ test). Also, maximum and minimum temperature showed a good agreement in both simulations when compared with observed data.

Keywords: RegCM4, physical parameters, dynamic vegetation, numerical modeling, remote sensing.

\section{Introdução}

Nos últimos séculos, com o advento da revolução industrial, as mudanças no uso e na cobertura da terra sofreram uma constante aceleração devido à globalização dos mercados, à expansão populacional e ao aumento da capacidade tecnológica. A substituição da cobertura natural ao longo do tempo tem provocado a diminuição no número de indivíduos, extinção, domesticação e mudanças de habitat de várias espécies (MEYER; TURNER, 1994).
Além disto, o desflorestamento, o uso de pastagens para criação de rebanhos, a expansão de áreas agrícolas e urbanas, a drenagem de áreas alagadas, entre outros usos, nunca se expandiram tão rapidamente em todo o mundo. Atualmente, grande parte das áreas do globo sofre algum tipo de manejo, que pode variar em vários graus de intensidade (RICHARDS, 1990).

Estima-se que nos últimos três séculos aproximadamente 1200 milhões de

\footnotetext{
* Graduado em Geografia pela Universidade do Estado de Santa Catarina (2004) e mestre em Sensoriamentp Remoto pelo Instituto Nacional de Pesquisas Espaciais (2008). Atualmente, é doutorando dos cursos de Sensoriamento Remoto (Inpe) e Geografia Física (USP). E-mail: gabriel@dsr.impe.be.

** E-mail: elisaiq@usp.br.
} 
hectares (Mha) de florestas e vegetação arbustiva tenham sido derrubadas e 560 Mha de gramíneas e pastagens, convertidas para outros usos e cobertura da terra. Nesse mesmo período, as áreas agrícolas em todo o globo aumentaram aproximadamente 1200 Mha, passando de 265 Mha no século XVIII (RICHARDS, 1990) para aproximadamente 1500 Mha nos dias atuais (FAO, 2002). Essas mudanças afetam significativamente o tempo e o clima (NIYOGI; MAHMOOD; ADEGOKE, 2009) e têm recebido atenção nas últimas décadas devido a seu papel na forçante radiativa global.

Dadas as circunstâncias acima descritas, as características físico-químicas e biológicas constituem-se em uma importante variável para a modelagem numérica de sistemas terrestres. Consequentemente, modelos de previsão do tempo, de clima e biogeoquímicos necessitam destes parâmetros para simular os processos físicos que ocorrem na biosfera-atmosfera (MCGUFFIE; HENDERSON-SELLERS, 2001). Todavia, em muitos modelos numéricos, os parâmetros físicos da vegetação estão defasados ou não variam em relação às estações seca e chuvosa, influenciando, desta forma, nos resultados obtidos a partir das simulações.

Nesse contexto, as imagens orbitais permitem estimar diversos parâmetros físico-químicos e biológicos (reflectância in-band, radiação fotossinteticamente ativa) e índices (vigor vegetativo e área foliar, entre outros) e podem ser utilizadas como dados de entrada para diversos modelos numéricos. Assim, o principal objetivo deste trabalho é verificar o impacto nas simulações numéricas do RegCM4 derivadas da atualização dos parâmetros físico-químicos e biológicos utilizados pelo Biosphere Atmosphere Transfer Scheme (Bats) como, por exemplo, os valores máximos e mínimos do índice de área foliar (IAF), os valores de reflectância no visível $(<0,7 \mu \mathrm{m})$ e no infravermelho próximo e médio ( $>, 7 \mu \mathrm{m}$ ) e fração máxima de cobertura vegetal.

\section{Material e método}

\section{1 modelo RegCM4 e os dados de entrada}

A primeira versão do modelo climático regional RegCM foi desenvolvida por Dickinson et al. (1989) e Giorgi (1989) no final da década de $80 \mathrm{com}$ base no modelo numérico Mesoscale Model versão 4 (MM4) e engloba vários esquemas de convecção cúmulos, umidade, condições iniciais e de contorno lateral, fluxos oceânicos e gradiente de pressão. Para as interações entre a atmosfera e a biosfera pode-se utilizar o modelo de superfície denominado Bats, que simula a influência da vegetação e umidade do solo no balanço de energia.

No Bats, estão representados 20 tipos de uso e cobertura da terra. Entre as principais informações utilizadas para os cálculos, destacam-se a textura e a cor dos solos (variando de grossa a fina; escura a clara) e a camada de neve, de solo e de raízes. As simulações nesse modelo incluem a determinação do conteúdo de água no solo, da condensação de umidade no dossel, da evapotranspiração, do escoamento superficial e da infiltração na camada de raízes (REGCM TEAM, 2010).

Neste trabalho, definiram-se como configurações do modelo as seguintes 
variáveis: (I) início da simulação no dia 1 de janeiro de 2007, final da simulação dia 1 de janeiro de 2008; (II) 180 pontos na coordenada x (longitude); (III) 180 pontos na coordenada y (latitude); (IV) 18 pontos em z (altitude); (V) resolução espacial de 50 $\mathrm{km}^{2}$, com início da grade em S $22^{\circ} 00^{\prime}$ e $\mathrm{O}$ $60^{\circ} 00^{\prime}$. Para as condições iniciais e de contorno lateral, assim como os dados de temperatura da superfície do mar (TSM), foram utilizados os dados provenientes do European Centre for Medium-Range Weather Forecasts (ECMWF), com resolução espacial de $1,5^{\circ}$ (aproximadamente $170 \times 170 \mathrm{~km}$ ), 37 níveis de pressão (para os dados meteorológicos). Neste trabalho, utilizaram-se os dados do ECMWF do ano de 2007.

\subsection{Material}

2.2.1 Dados de precipitação e temperatura do ar

Para avaliar as simulações realizadas no RegCM4, utilizaram-se as séries históricas de precipitação $(\mathrm{mm})$, temperatura mínima $\left({ }^{\circ} \mathrm{C}\right)$ e temperatura máxima $\left({ }^{\circ} \mathrm{C}\right)$ disponibilizada pelo CPTEC. Estes dados correspondem às informações derivadas do Surface Synoptic Observations (SYNOP/INMET), das plataformas de coleta de dados (PCDs) do Centro de Missão de Coleta de Dados do Instituto Nacional de Pesquisas Espaciais (CMCD/Inpe) e parceiros do Programa de Monitoramento de Tempo, Clima e Recursos Hídricos (PMTCRH/INPE).

Os dados do Tropical Rainfall Measuring Mission (TRMM) foram utilizados neste trabalho para analisar a distribuição espacial da precipitação sobre a América do Sul e verificar a consistência dos resultados de precipitação obtidos com o RegCM4. O satélite TRMM foi desenvolvido através de uma parceria entre a National Aeronautics and Space Administration (Nasa) e a agência espacial japonesa Japan Aerospace Exploration Agency (Jaxa). De modo geral, o lançamento do satélite TRMM em novembro de 1997 permitiu a obtenção de informações sobre as características da precipitação em lugares que têm baixa densidade de redes de estações meteorológicas como, por exemplo, os estados do Acre, Amapá, Mato Grosso, Mato Grosso do Sul, Pará, Rondônia e Roraima, entre outros. Os dados utilizados neste trabalho foram extraídos do produto 3B43 V6, com uma resolução temporal mensal, cobertura geográfica correspondente a $50^{\circ} \mathrm{S}$ a $50^{\circ} \mathrm{N}$ e resolução espacial de $0,25^{\circ}$ $x \quad 0,25^{\circ} \quad$ (disponibilizado em http://mirador.gsfc.nasa.gov/).

2.2.2 Produtos do sensor Moderate Resolution Imaging Spectroradiometer (Modis)

Neste trabalho, utilizaram-se os produtos MCD12Q1 (uso e cobertura da terra para a América do Sul, resolução espacial de $500 \times 500 m$, anual), MCD12C1 (uso e cobertura da terra, resolução espacial de $5.600 \times 5.600 \mathrm{~m}$, global e anual), MOD13C2 (índice de vegetação, resolução espacial de $5.600 \times 5.600 \mathrm{~m}$, global e mensal), MCD15A2 (índice de área foliar - IAF, resolução espacial de $1.000 \times 1.000 \mathrm{~m}$, por regiões e a cada 8 dias), MOD16A3 (evapotranspiração, resolução espacial de $1.000 \times 1.000 \mathrm{~m}$, global e anual) e MCD43C3 (albedo, resolução espacial de $5.600 \times 5.600$ m, global e a cada 16 dias) disponíveis em <https://wist.echo.nasa.gov/api/> e exemplificados na Figura 1. 
Figura 1 - Área de estudo contendo: (a) índice de vegetação mensal estimado pelo produto MOD13C2 para julho de 2007; (b) mosaico de 16 dias para o albedo da superfície (4 a 20 de julho de 2007); e (c) mosaico de 8 dias do índice de área foliar (IAF) entre 12 e 20 de julho de 2007

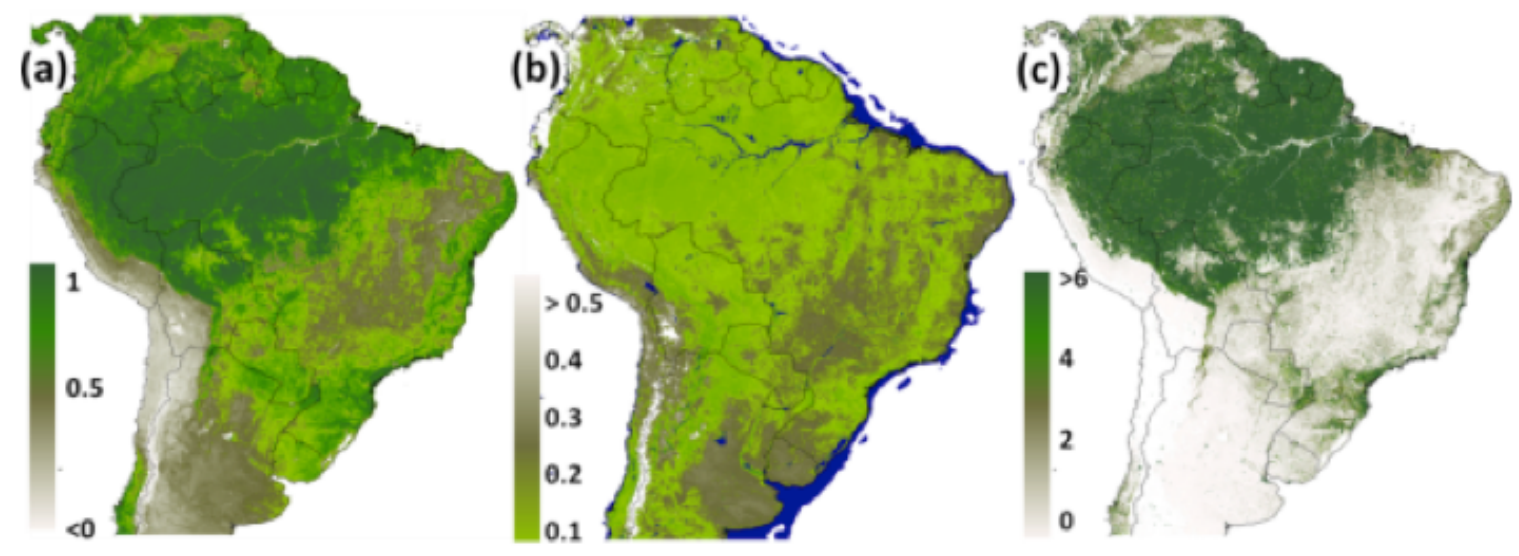

\subsection{Metodologia}

2.3.1 Alteração dos parâmetros físicos utilizados pelo Bats

Atualmente, o mapa de uso e cobertura da terra implementado no RegCM4, e utilizado pelo modelo de superfície Bats, utiliza os dados provenientes do Global Land Cover Characterization (GLCC) referentes ao ano de 1992. Consequentemente, usaram-se os dados de uso e cobertura da terra provenientes do produto MCD12Q1 (para a América do Sul) e MCD12C1 (para o restante do globo) para a atualização do mapa para o ano de 2007. Nessa etapa, usou-se o esquema de classificação proposto pelo International

Geosphere-Biosphere Programme (IGBP) que separa a superfície em 17 classes.

O Bats utiliza, para o cálculo dos processos que ocorrem na superfície, uma tabela de parâmetros que contém as informações da fração máxima de cobertura vegetal, textura e cor do solo, rugosidade, valor máximo e mínimo de IAF, tamanho da folha, profundidade do solo, reflectância inband para comprimentos de onda menores que $0,7 \mu \mathrm{m}$, reflectância in-band para comprimentos de onda maiores que 0,7 $\mu \mathrm{m}$, entre outros. Desta forma, para cada classe de uso e cobertura da terra, estes valores são utilizados para resolver as equações físicas. Uma das limitações dessa tabela é a invariância das propriedades físico-químicas e biológicas da cobertura vegetal ao longo das estações do ano.

Para atualizar os principais parâmetros físicos (fração máxima de cobertura vegetal, valor máximo e mínimo de IAF, reflectância in-band para comprimentos de onda menores que 0,7 $\mu \mathrm{m}$ e reflectância in-band para comprimentos de onda maiores que $0,7 \mu \mathrm{m}$ ) foram utilizados diversos produtos orbitais derivados do sensor Modis/Aqua-Terra. Consequentemente, para cada classe de uso e cobertura da terra foram extraídos os valores médios anuais para a vegetação na América do Sul. 
2.3.2 Estimativas da fração máxima de cobertura vegetal

O índice de vegetação por diferença normalizada (NDVI) é utilizado para medir o vigor vegetativo e monitorar a cobertura vegetal de uma determinada superfície a partir das informações espectrais obtidas pelos satélites. O NDVI é estimado pela seguinte razão (ROUSE et al., 1974):

$$
\text { NDVI }=\frac{\rho_{\text {ivp }}-\rho_{\text {vermelho }}}{\rho_{\text {ivp }}+\rho_{\text {vermelho }}}
$$

em que $\rho_{i v p}$ representa a reflectância in-band na faixa do espectro eletromagnético (EEM) referente ao infravermelho próximo $(0,73$ $\mu \mathrm{m}-1,3 \mu \mathrm{m})$ e $\rho_{\text {vermelho }}$ representa a faixa do EEM referente ao vermelho $(0,622 \mu \mathrm{m}-0,700$ $\mu \mathrm{m})$. Ressalta-se que a faixa do EEM imageada pelos sensores pode variar de sensor para sensor. Com base no NDVI podese estimar a fração de cobertura vegetal $\left(\sigma_{v}\right)$ a partir de três metodologias diferentes.

De modo geral, cada pixel que compõe uma imagem orbital pode ser representado por uma média ponderada da radiância espectral de cada elemento. Essa mistura se deve à existência de mais de um alvo na área de abrangência de cada pixel, em que o valor digital tem informações sobre a proporção da mistura de cada alvo (ROBERTS et al., 1998). Consequentemente, a resposta espectral dos pixels em qualquer comprimento de onda pode ser considerada uma combinação de cada componente da mistura (SHIMABUKURO; SMITH, 1991).

Considerando o nível digital uma média ponderada entre os elementos que compõem o pixel, a radiância espectral estimada pelo sensor $\left(L_{\lambda}\right)$ pode ser definida como o somatório linear das componentes da vegetação $\left(\sigma_{v}\right)$ e do solo exposto $\left(1-\sigma_{v}\right)$ :

$$
L_{\lambda}=b_{\lambda, v}+{ }_{v}^{1-}\left(\phi \quad L_{v}\right)_{\lambda, s}
$$

em que $L_{\lambda, v}$ representa a radiância espectral da vegetação e $L_{\lambda, s}$ a radiância espectral do solo. Aplicando essa relação linear no NDVI, pode-se determinar a fração de cobertura vegetal a partir de:

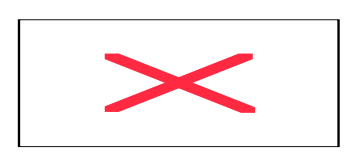

Embora alguns estudos apontem uma relação não linear entre o NDVI e a fração de cobertura vegetal (CARLSON; RIPLEY, 1997), outros propõem que a abordagem linear para estudos globais é adequada, sobretudo devido à falta de dados necessários à solução das equações não lineares. Assim, a equação 3 será utilizada neste trabalho para determinar a fração máxima de cobertura vegetal a partir dos dados de NDVI estimados pelo sensor Modis/Terra.

Para a configuração do modelo, relacionadas à parametrização dos processos físicos, foram empregados os métodos mais utilizados na literatura. Desta forma, utilizouse a técnica de relaxamento exponencial para o contorno lateral do modelo, e os seguintes esquemas: (I) fechamento cúmulos desenvolvido por Fritsch e Chappell (1980); (II) fluxo oceânico proposto por Zeng, Zhao e Disckinson (1998); (III) condições iniciais proposto por Holtslag, De Bruijn e Pan (1990); e (IV) dedução hidrostática com perturbação da temperatura para o gradiente de pressão. A partir dessas configurações, a simulação foi baseada nas alterações da fração máxima de cobertura vegetal, IAF máximo e mínimo, reflectância in-band para 
comprimentos de onda menores que 0,7 $\mu \mathrm{m}$ e reflectância in-band para comprimentos de onda maiores que 0,7 $\mu \mathrm{m}$.

\section{Resultados e discussão}

A Figura 2 mostra a variação trimestral (janeiro-fevereiro-março; abril-maio-junho; julho-agosto-setembro; outubro-novembro-dezembro; JFM, AMJ, JAS, OND, respectivamente) da fração máxima de cobertura vegetal (FCV), e é possível ver a dinâmica da vegetação e sua variação fenológica ao longo do ano. Entre os meses de janeiro e março, época chuvosa em praticamente todo o Brasil, verificam-se valores elevados de FCV em todas as regiões (Figura 2a). Consequentemente, à medida que o volume de precipitação começa a diminuir no Brasil Central e na Patagônia argentina, os valores de FCV vão diminuindo gradativamente (Figura 2b). Entre os meses de julho e setembro, estação seca em grande parte da América do Sul, verifica-se uma queda acentuada nos valores de FCV (Figura 2c), que, em alguns casos, podem cair de 0,8 para menos de 0,20 (como se vê no Nordeste do Brasil). Ainda, com a transição da estação seca para a chuvosa (Figura 2d), os valores de FCV começam a aumentar novamente.

Figura 2 - (a) Valores de FCV para os meses de janeiro, fevereiro e março (JFM); (b) Valores de FCV para os meses de abril, maio e junho (AMJ); (c) Valores de FCV para os meses de julho, agosto e setembro (JAS); e (d) Valores de FCV para os meses de outubro, novembro e dezembro (OND)

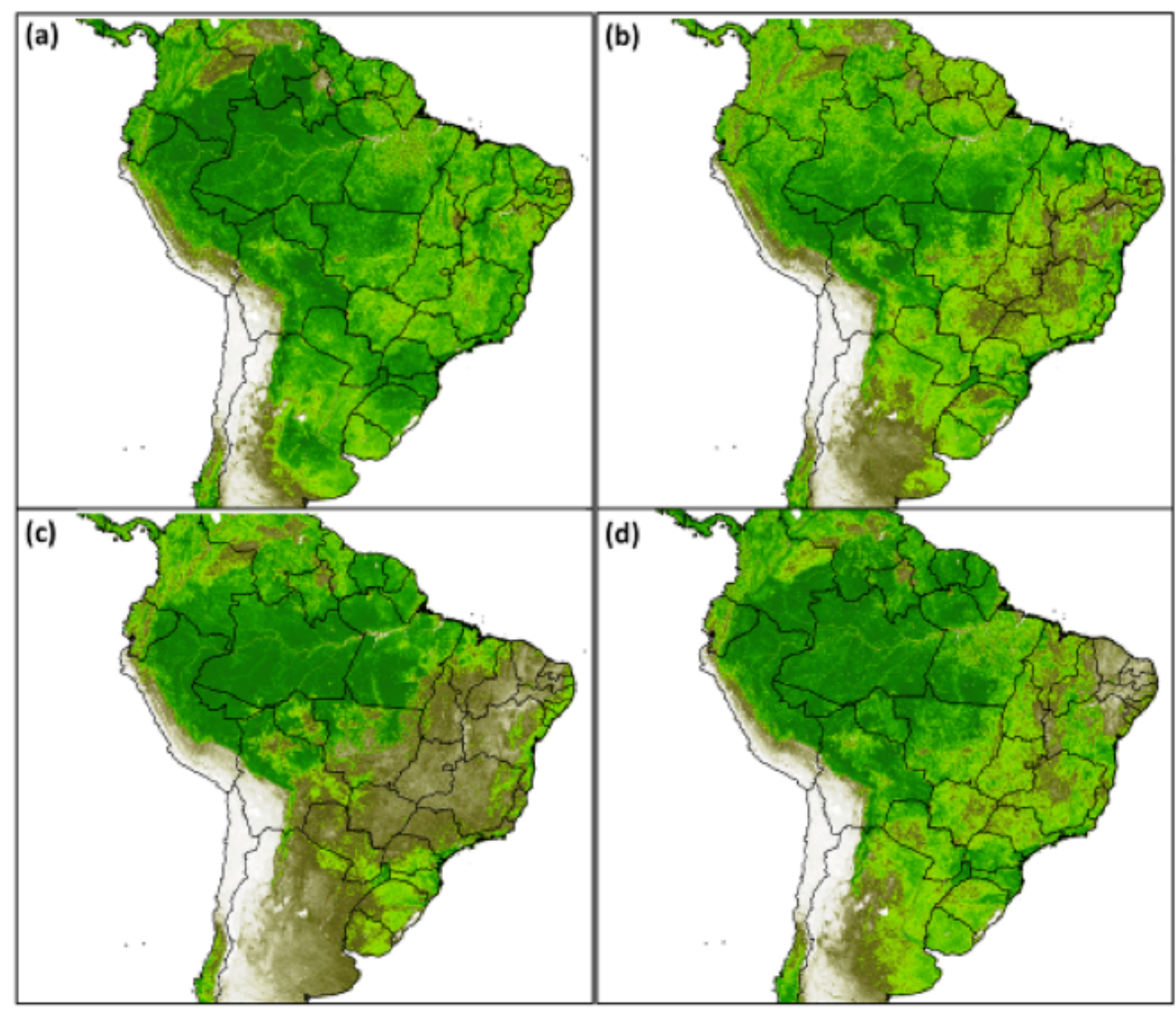


A variação fenológica da vegetação ao longo do ano modifica a interação da radiação eletromagnética com a superfície terrestre (Figura 3), assim como o balanço de energia. Além disso, variações significativas na disponibilidade de água alteram a evapotranspiração e a formação de nuvens, entre outros fatores, influenciando significativamente as variáveis atmosféricas como, por exemplo, temperatura do ar, calor latente e sensível e precipitação, entre outros. Na Figura 3a, verifica-se que em grande parte do Brasil, da Argentina e da Bolívia, os valores de albedo aproximam-se de $24 \%$, mas, com o início da estação seca e, consequentemente, com a perda de vigor vegetativo, os valores decaem, aproximandose de $15-18 \%$ (Figuras 3b e 3c). Ainda, com o decorrer da transição da estação seca para a chuvosa em todo o Brasil, esses valores começam a subir novamente, principalmente no Brasil central, favorecendo o processo agrícola.

Dadas essas circunstâncias, no modelo de superfície Bats, os parâmetros físicos da vegetação são estáticos, ou seja, não variam ao longo do ano. Assim, neste trabalho, optou-se por fazer duas simulações no RegCM4, para avaliar a resposta do modelo a partir da dinâmica da vegetação, compreendendo: (I) alteração dos parâmetros físicos a cada três meses (índice de área foliar - IAF, reflectância no infravermelho próximo e médio - RVIVPM, FCV); II) rodada original sem alterações no uso e cobertura da terra e nas propriedades físico-químicas e biológicas.

Figura 3 - Variação trimestral para o ano de 2007 do albedo da superfície para os trimestres: (a) JFM, (b) AMJ, (c) JAS e (d) OND

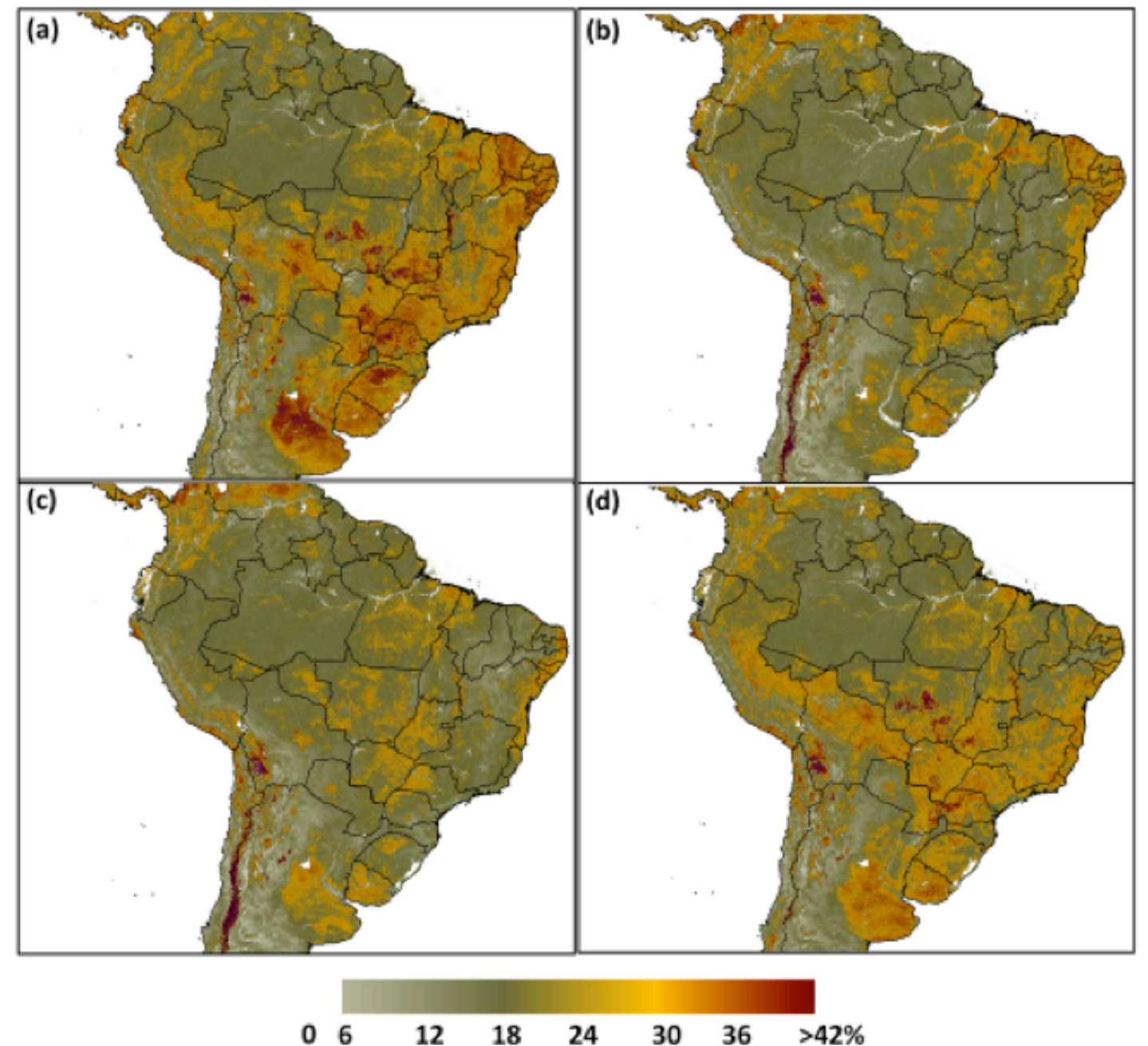


Para cada simulação, adotaram-se siglas referentes para o modelo de superfície (Bats, B), o mapa de uso e a cobertura da terra utilizada (original, UO; alterado, UA), o dado de TSM (Era-interim, SE), as condições iniciais e de contorno lateral (Era-interim, EI), o esquema de parametrização de cúmulos (Emmanuel, EM), e as alterações temporais nos parâmetros físicos no Bats (BA-FCV-IAF-REF-TMP). A Figura 4 exibe a precipitação anual correspondente à simulação inicial com o mapa de uso e a cobertura da terra original do modelo (para o ano de 1992) (Figura 4a, B_UO_SE_EI_EM), a precipitação anual simulada no modelo RegCM4 a partir da variação trimestral dos parâmetros físicos referentes à fração máxima de cobertura vegetal (FCV), ao índice de área foliar (IAF), à reflectância no visível e no infravermelho próximo e médio (REF) e com a parametrização cúmulos de Emmanuel (1991) (Figura 4b) e o mapa de precipitação para o ano de 2007 estimado pelo TRMM (Figura 4c).

A alteração trimestral das características físicas da vegetação ocasionou uma alteração significativa do padrão de distribuição da precipitação para o ano de 2007. Entre as principais diferenças entre as simulações (Figuras 4a e 4b) destacam-se o aumento da precipitação na região Sul, principalmente no Oeste de Santa Catarina. Além disso, houve uma redução significativa na precipitação na região de atuação da ZCAS, principalmente nos estados de Goiás, Minas Gerais e Mato Grosso. Ainda, pode-se destacar uma redução na precipitação da região Amazônica. De um modo geral, as maiores diferenças são encontradas em áreas na qual há uma elevada taxa de precipitação. A alteração das características físicas da vegetação e do mapa de uso e cobertura da terra ocasionou uma redução significativa dos valores de precipitação para os estados de Minas Gerais, Bahia, Espírito Santo, na região central de Goiás e em regiões próximas à cordilheira dos Andes. Ainda, nota-se uma redução na região norte do Mato Grosso devido principalmente à alteração da classe floresta ombrófila densa para áreas de pastagem e agricultura. 
Figura 4 - (a) Precipitação anual para o ano de 2007 estimada a partir das condições originais do modelo RegCM4 (B_UA_SE_EI_EM); (b) Precipitação anual para o ano de 2007 estimada a partir da variação trimestral dos parâmetros físicos (B_UA_SE_EI_EM_BA-FCV-IAF-REF-TMP); (c) Mapa anual de precipitação estimada pelo TRMM para o ano de 2007
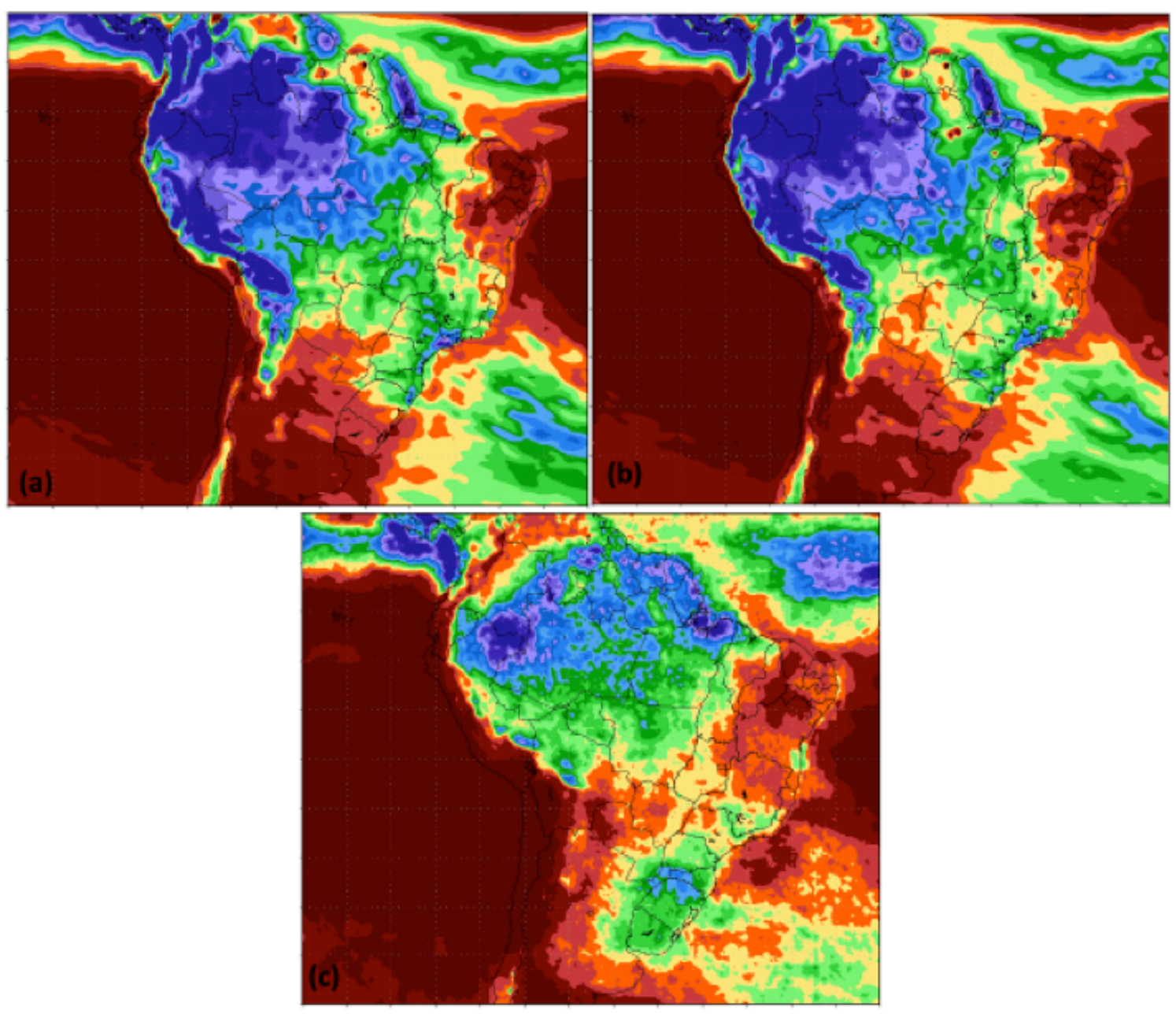

$\begin{array}{llllllll}0 & 600 & 1200 & 1800 & 2400 & 3000 & 3600 & 4200 \\ \text { mmano }\end{array}$

A Figura $5 a$ exibe 0 gráfico de dispersão resultante da comparação entre a simulação para o ano de 2007 no RegCM4 utilizando os dados de entrada originais do modelo e os dados estimados pelo TRMM. Nesta, é possível verificar que os dados de precipitação são subestimados em 12\%, quando comparados com os dados estimados por satélite, com uma correlação de $84 \%$ (significante a $\mathrm{p}<0,05$, teste t-student). Assim, introduzindo a variação trimestral dos parâmetros físicos mencionados acima, a precipitação estimada pelo modelo, indicam uma redução significativa do erro (subestimativa de 3\%, correlação de 92\%, significante a $\mathrm{p}<0,05$, teste t-student). Em relação à temperatura máxima e mínima, percebe-se que em ambas as simulações apresentam uma boa concordância com os dados observados. Em geral, as temperaturas máximas mensais apresentaram uma correlação de aproximadamente 95\% e valores simulados próximos ao esperado (significante a $\mathrm{p}<0,05$, teste t-student). 
Ainda, as temperaturas mínimas simuladas pelo RegCM4 apresentaram correlações de $97 \%$ (significante a $p<0,05$, teste $t-$ student) e valores muito próximos aos observados pela rede de estações meteorológicas do INMET e CPTEC.

Figura 5 - Comparação entre os dados de precipitação provenientes do TRMM e de temperatura máxima e mínima coletadas pelas 183 estações meteorológicas distribuídas pelo Brasil $e$ as simulações realizadas no RegCM4 a partir das condições originais do modelo ( $a, c, e)$ e com as alterações na parametrização de superfície $(b, d, f)$

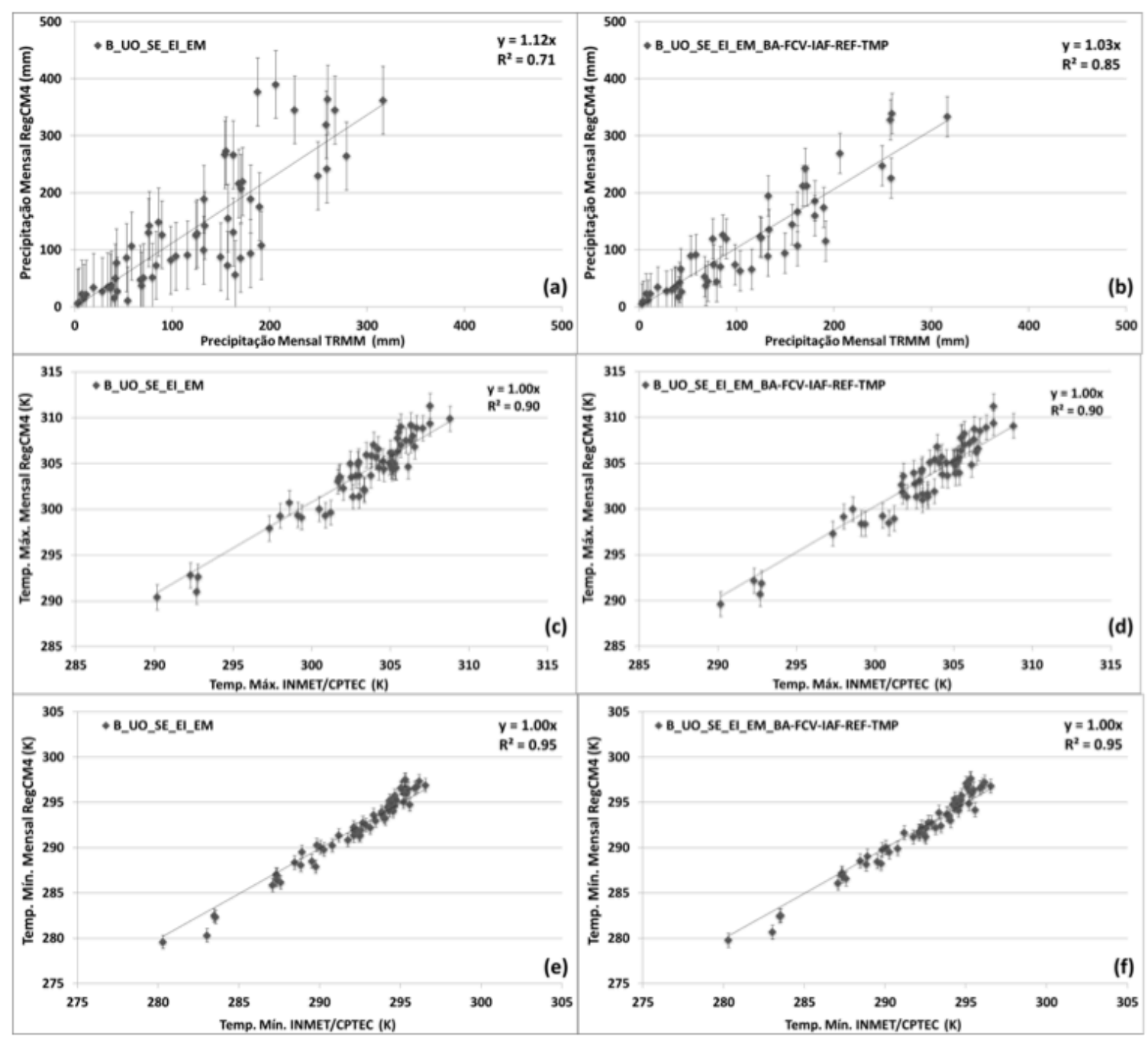

\section{Considerações finais}

O uso e cobertura da terra que compõem a superfície terrestre controlam as interações da atmosfera com as radiações solar e termal, devido à influência de suas características físico-químicas e biológicas sobre a atmosfera. Entretanto, é comum encontrar modelos regionais e globais na qual o mapa de uso e cobertura da terra encontrase defasados no tempo, influenciando, desta forma, nos resultados das simulações. Consequentemente, dados de sensoriamento remoto provenientes de plataformas orbitais possibilitam a obtenção de informações multiespectrais com variadas resoluções espaciais e temporais. 
A partir dos aprimoramentos realizados no RegCM4, foi possível constatar que a variação trimestral dos parâmetros físicos utilizados pelo modelo de superfície Bats reduziram para $3 \%$ as superestimativas de precipitação para a área das estações meteorológicas, com uma correlação de $92 \%$ (significante a $\mathrm{p}<0,05$, teste t-student). Ainda, a simulação das temperaturas mensais máximas e mínimas apresentaram uma correlação de aproximadamente 95\% e valores simulados próximos ao esperado, indicando uma boa consistência do modelo.

\section{Agradecimento}

À Fundação de Amparo à Pesquisa do Estado de São Paulo (Fapesp) pelo apoio (2010/07083-0 e 2010/17437-4).

\section{Referências bibliográficas}

CARLSON, T. N.; RIPLEY, D. A. On the relation between NDVI, fractional vegetation cover, and leaf area index. Remote Sensing of Environment, v. 62, p. 241-252, 1997.

DICKINSON, R. E. et al. A regional climate model for the western United States. Climatic Change, n. 15, p. 383-422, 1989.

FAO. World agriculture towards 2015/30. Summary report. Rome: FAO, 2002.

FRITSCH, J. M.; CHAPPELL, C. F. Numerical prediction of convectively driven mesoscale pressure systems. Part i: Convective parameterization.

Journal of Atmospheric Sciences, n. 37, p. 1722-1733, 1980.

GIORGI, F. Two-dimensional simulations of possible mesoscale effects of nuclear war fires. Journal of Geophysical Research, v. 94 , n. 1, p. 11451163, 1989.

HOLTSLAG, A. A. M.; DE BRUIJN, E. I. F.; PAN, H.-L. A high resolution air mass transformation model for short-range weather forecasting. Monthly Weather Review, n. 118, p. 15611575, 1990.

MCGUFFIE, K.; HENDERSON-SELLERS, A. Forty years of numerical climate modelling. International Journal of Climatology, v. 21, n. 9, p. 10671109, 2001.

MEYER, W. B.; TURNER, B. L. Changes in Land Use and Land Cover: A Global Perspective. Cambridge: Cambridge University Press, 1994.

NIYOGI D.; MAHMOOD, R.; ADEGOKE, J. O. Land-use/land-cover change and its impacts on weather and climate. Boundary-Layer Meteorology, v. 133, p. 297-298, 2009.

REGCM TEAM. RegCM Version 4.0 Core Description. Disponível em: <http://eforge.esciencelab.org/gf/download/docmanfileversio n/4/235/regcm-guide.pdf>. Acesso: 18 ago. 2011.

RICHARDS, J. F. Land transformation. In: TURNER II, B. L. et al. (Eds.). The Earth as Transformed by Human Action. New York: Cambridge University Press, 1990. p. 163-178.

ROBERTS, D. A. et al. Change identification using multitemporal spectral mixture analysis: applications in eastern Amazonia. In: LUNETTA, R. S.; ELVIDGE, C. D. (Eds.). Remote sensing change detection: environmental monitoring methods and applications, v. 1. Michigan: Ann Arbor Press, 1998. p. 137-161.

ROUSE, J. W. et al. Monitoring the vernal advancement of retrogradation of natural vegetation. Final Report. Greenbelt, MD: Nasa/GSFC, 1977.

SHIMABUKURO, Y. E.; SMITH, J. A. The least squares mixing models to generate fraction images derived from remote sensing multispectral data. IEEE Transactions on Geoscience and Remote Sensing, v. 29, n. 1, p. 1620, 1991.

ZENG, X.; ZHAO, M.; DICKINSON, R. E. Intercomparison of bulk aerodynamic algoriths for the computation of sea surface fluxes using toga coare and tao data. Journal of Climate, v. 11 , p. 2628-2644, 1998. 